\title{
PRDX6 promotes proliferation and induces chemo-resistance via peroxidase activity in Toledo diffuse large B-cell lymphoma cells
}

\author{
Zhigang Fang ${ }^{1 \#}$, Tingrong Liu ${ }^{2 \#}$, Xiangfu Liu ${ }^{3 \#}$, Ying Lu ${ }^{3}$, Yanling Sun ${ }^{1}$, Ruozhi Xiao ${ }^{1}$, Ruifang Fan ${ }^{4}$, \\ Lingling Liu ${ }^{1}$ \\ ${ }^{1}$ Department of Hematology, The Third Affiliated Hospital of Sun Yat-sen University \& Sun Yat-sen Institute of Hematology, Guangzhou 510630, \\ China; ${ }^{2}$ Department of Cardiology, The Third Affiliated Hospital of Southern Medical University, Guangzhou 510630, China; ${ }^{3}$ Department of \\ Blood Transfusion, ${ }^{4}$ Prevention Department of Health, The Third Affiliated Hospital of Sun Yat-sen University, Guangzhou 510630, China \\ Contributions: (I) Conception and design: L Liu, R Fan; (II) Administrative support: L Liu; (III) Provision of study materials or patients: Z Fang, T \\ Liu; (IV) Collection and assembly of data: X Liu; (V) Data analysis and interpretation: Y Lu, Y Sun, R Xiao; (VI) Manuscript writing: All authors; (VII) \\ Final approval of manuscript: All authors. \\ \#These authors contributed equally to this work. \\ Correspondence to: Lingling Liu. Department of Hematology, The Third Affiliated Hospital of Sun Yat-sen University, No. 600 Tianhe Road, \\ Guangzhou 510630, China. Email: liull35@mail.sysu.com.cn; Ruifang Fan. Prevention Department of Health, the Third Affiliated Hospital of Sun \\ Yat-sen University, No. 600 Tianhe Road, Guangzhou 510630, China. Email: xfangcn@163.com.
}

Background: Diffuse large B cell lymphoma (DLBCL) is the most common type of non-Hodgkin lymphomas. Despite the application of rituximab, cyclophosphamide, doxorubicin, vincristine, and prednisone (R-CHOP) regimen being effective on 70-80\% of DLBCL patients, the remaining 20-30\% develop an even more aggressive relapsed tumor. PRDX6 has been shown to play important roles in multiple cancers. However, there is no study about the role of PRDX6 in DLBCL.

Methods: The stable Toledo cell lines that overexpression or knockdown of PRDX6 gene were established. Western blot was used to determine the quantity of PRDX6 protein. Then, the function of PRDX6 in Toledo cell proliferation was determined using cell counting assay and Annexin V/PI analysis assays, and the underlying mechanism was determined through glutathione peroxidase activity and iPLA2 activity assay.

Results: In the current study, we showed that the expression of PRDX6 was critical for the proliferation of Toledo DLBCL cells. Additionally, knockdown of PRDX6 induced apoptosis in Toledo DLBCL cells. Importantly, overexpression of PRDX6 caused a doxorubicin resistance in Toledo DLBCL cells, while downregulation of PRDX6 significantly enhanced doxorubicin induced apoptosis. Interestingly, the glutathione peroxidase activity of PRDX6, but not the phospholipase A2 activity, was crucial for PRDX6 induced proliferation and anti-apoptosis effects. Together, our study explored the tumor promoting function of PRDX6 in DLBCL for the first time.

Conclusions: Our data indicated that PRDX6 could be a target for overcoming drug resistance. Targeting PRDX6 expression or peroxidase activity could be an effective strategy to overcome drug resistance in clinical DLBCL treatment.

Keywords: PRDX6; peroxidase activity; chemo-resistance; diffuse large B cell lymphoma (DLBCL)

Submitted Apr 03, 2018. Accepted for publication Aug 13, 2019.

doi: $10.21037 /$ tcr.2019.08.36

View this article at: http://dx.doi.org/10.21037/tcr.2019.08.36 


\section{Introduction}

Diffuse large B cell lymphoma (DLBCL) is the most common type of B cell non-Hodgkin's lymphoma (NHL), and accounts for $30-40 \%$ of NHL cases (1). The combined chemotherapy of rituximab, cyclophosphamide, doxorubicin, vincristine, and prednisone (R-CHOP) has increased DLBCL survival substantially, with complete remission (CR) in approximately $80 \%$ patients (2). Although tremendous progress has been made in the outcomes of DLBCL, 30-40\% of patients are refractory to treatment or relapse after initial response to therapy (3). Thus, investigating the molecular mechanisms underlying DLBCL chemo-resistance is critical for improving the treatment response.

Peroxiredoxins (PRDX) family contains six members of peroxidase (PRDX1-PRDX6). PRDX6 is a unique member of PRDXs family which displays both glutathione peroxidase and phospholipase A2 activities (4). The glutathione peroxidase activity of PRDX6 is crucial for reducing cellular $\mathrm{H}_{2} \mathrm{O}_{2}$ levels and decreasing oxidative stress-induced lipid peroxidation, membrane damage, and apoptosis (5-7). The phospholipase A2 activity of PRDX6 catalyzes the hydrolysis of the sn-2 fatty acyl ester bond of glycerophospholipids to produce free fatty acids and lysophospholipids, and is critical in phospholipid metabolism and cell signaling (8). Recent evidence suggests that PRDX6 plays important roles in multiple cancers. For example, PRDX6 is upregulated in cancerous hepatoma cell line compared with noncancerous counterpart. The anti-oxidant activity of PRDX6 suppresses peroxideinduced cytotoxicity in hepatoma cell (9). Additionally, the phospholipase A2 activity of PRDX6 is important for PRDX6 promoted proliferation and induction of Src family kinase activation in melanoma cells (10). Moreover, PRDX6 also promotes invasion and metastasis in lung cancer cells via its phospholipase A2 activity (11). However, little is known about the role of PRDX6 in DLBCL, and the underlying molecular mechanism is largely unknown.

In current study, we evaluate the functions of PRDX6 in DLBCL, and demonstrate the potential molecular mechanism behind it. We found that overexpression of PRDX6 significantly promoted proliferation of Toledo DLBCL cells, while downregulation of PRDX6 suppressed the proliferation. Additionally, downregulating the expression of PRDX6 induced apoptosis in Toledo DLBCL cells. Importantly, our data showed that upregulation of PRDX6 alleviated doxorubicin induced apoptosis, while downregulation of PRDX6 produced a synergistic effect on apoptosis when Toledo DLBCL cells treated with doxorubicin. Mechanically, PRDX6 displayed both glutathione peroxidase and phospholipase A2 activities in Toledo DLBCL cells. Interestingly, inhibition of glutathione peroxidase activity of PRDX6 by M-succinate (mercaptosuccinate) treatment reversed PRDX6 promoted proliferation and anti-apoptosis effects, while inhibition of phospholipase A2 activity did not. Our data indicated that PRDX6 was a critical molecule to induce doxorubicin resistance, targeting the glutathione peroxidase activity of PRDX6 could be a promising strategy to overcome doxorubicin resistance.

\section{Methods}

\section{Reagents and cells culture}

Doxorubicin and Annexin V-FITC Apoptosis Detection Kit were purchased from Sigma-Aldrich. Flag, PRDX6, and GAPDH were purchased from Cell Signaling Technology. Toledo was obtained from the American Type Culture Collection (ATCC) and was cultured in RPMI-1640 (Gibco) supplemented with $10 \%$ fetal bovine serum (FBS; Hyclone). Cells were maintained at $37{ }^{\circ} \mathrm{C}$ in a humidified $5 \% \mathrm{CO}_{2}$ atmosphere.

\section{shPRDX6 stable cell lines established}

Knockdown of gene was performed with the specific shRNA delivered by a lentiviral system purchased from Sigma-Aldrich Corp, according to the instruction manual. 293T cells were co-transfected with pMD2. $\mathrm{G}$ and psPAX2 compatible packaging plasmids and pLKO.1 plasmid bearing the specific shRNA for $24 \mathrm{~h}$. The cultured medium containing lentivirus was collected. Then Toledo cells were infected with the lentivirus bearing the shRNA in growth medium containing $8 \mu \mathrm{g} / \mathrm{mL}$ polybrene for $24 \mathrm{~h}$. Afterwards, cells were subcultured and selected with $2 \mu \mathrm{g} / \mathrm{mL}$ puromycin. The shRNA constructs targeting the gene and referring to the sequence is: PRDX6 (NM_004905.2): TRCN0000052154 (5'-CCGGCCGAAAGGAGTCTTCACCAAACTCGAG TTTGGTGAAGACTCCTTTCGGTTTTTG-3').

\section{PRDX6-flag overexpressed stable cell lines established}

The plasmids encoding PRDX6 was generated by PCR amplification and subcloned into the pLVX-DsRed- 
Monomer-N1 expression vector. The primers for gene PRDX6-flag cloning were as follow: 5'-AACTCGAGAT GCCCGGAGGTCTGCTTCTC-3'; 5'-AAGAATTC TTACTTATCGTCGTCATCCTTGTAATCAGGCT GGGGTGTGTAGCGGAG-3'. 5×106 293T cells were transfected with lentiviral vector, psPAX2 and pMD2. G. Supernatants were collected every $24 \mathrm{~h}$ between 24 to $72 \mathrm{~h}$ after transfection, pulled together and concentrated via ultracentrifugation, and the viral titer was determined by serial dilutions. The multiplicity of infection during transfection was $10.2 \times 10^{5}$ cell were treated with polybrene $(8 \mu \mathrm{g} / \mathrm{mL})$ and virus contained supernatants for $24 \mathrm{~h}$. Cells were growth for another $48 \mathrm{~h}$. Cells were treated with puromycin $(2 \mu \mathrm{g} / \mathrm{mL})$ for $72 \mathrm{~h}$ to establish stable cells.

\section{Small interfering $R N A$ transfection}

Toledo cells were seeded in six-well plates. In each well, $100 \mathrm{nM}$ of siRNA and $5 \mu \mathrm{L}$ of Lipofectamine 2000 were added to Opti-MEM, mixed and then added to the cells. After transfection of siRNAs for 24 or $48 \mathrm{~h}$, RNAi efficiency was determined by western blot. The siRNA was purchased from GenePharma company and the sequence was as follow: si-PRDX6 5'-CCGAAAGGAGTCTTCACCAAA-3'.

\section{Cell counting assay}

About $1 \times 10^{5}$ cells per well were plated in six-well plates. Subsequently, cells were treated. Then, cell count was determined with trypan blue exclusion assay.

\section{Annexin V/PI analysis}

Cells treated with indicated concentration of indicated drug for indicated time. Cells were collected and resuspended in the binding buffer (500 $\mu \mathrm{L} /$ sample). Annexin-V-FITC ( $5 \mu \mathrm{L} /$ sample) was added to the cells followed by addition of $5 \mu \mathrm{L} /$ sample PI (propidium iodide). The samples were then incubated for $15 \mathrm{~min}$ in the dark at $4{ }^{\circ} \mathrm{C}$ and subjected to flow cytometry analysis (12).

\section{Western blot analysis}

Cells were harvested and lysed in RIPA buffer. The protein concentration was determined by Bradford method with BSA (Sigma) as the standard. Equal amounts of cell extract were subjected to electrophoresis in SDS-polyacrylamide gel and transferred to nitrocellulose membrane (Millipore).
The membranes were blocked and then incubated with flag, PRDX6 and GAPDH (Cell Signaling Technology Corp, Beverly, MA, USA) antibodies at $4{ }^{\circ} \mathrm{C}$ overnight, followed by incubation for $1 \mathrm{~h}$ room temperature with appropriate secondary antibodies. Antibody binding was detected with an enhanced chemiluminescence kit (Pierce).

\section{Glutatbione peroxidase activity and iPLA2 activity assay}

Glutathione peroxidase assay (No. 703102) and cPLA2 assay kits (No. 765021) were purchased from Cayman Chemical and iPLA2 and glutathione peroxidase activities were measured according to the manufacturer's recommendations (13).

\section{Statistics}

Statistical analysis was performed using SPSS version 16.0 (SPSS Inc.) and GraphPad Prism 6 (GraphPad Software, Inc.). The Student's $t$ test and multi-factorial ANOVA were used to make a statistical comparison. The level of significance was set at $\mathrm{P}<0.05$.

\section{Results}

\section{PRDX6 is critical for cell proliferation in Toledo DLBCL cells}

To study the functions of PRDX6 in DLBCL, we evaluated the proliferation of Toledo DLBCL cells at first. We established a stable PRDX6-flag overexpressed cell line (PRDX6 cells) and the control cell line (Vec cells, vector cell) in Toledo DLBCL cells by lentivirus mediated gene transfer. Western blot analysis showed that PRDX6 cells indeed overexpressed PRDX6-flag protein (Figure 1A). We then evaluated proliferation by cell counting assay. As shown in Figure 1B, PRDX6 cells displayed a significant advantage in cell proliferation as compared with Vec cells. To further confirm the effect on proliferation, we established another stable cell line expressed PRDX6 shRNA (shPRDX6 cells) and the control cell line (NC cells, negative control cells) in Toledo DLBCL cells by lentivirus mediated gene transfer. The expression of PRDX6 was down regulated up to approximately $70 \%$ in shPRDX6 cells as compared with NC cells (Figure 1B). The proliferation of Toledo DLBCL cells was significantly suppressed by downregulation of PRDX6 (Figure 1B). These data indicated that PRDX6 has an important role in regulating the proliferation of Toledo DLBCL cells. 
A

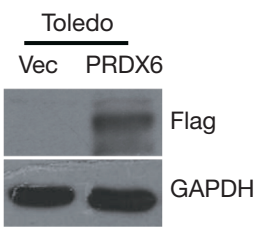

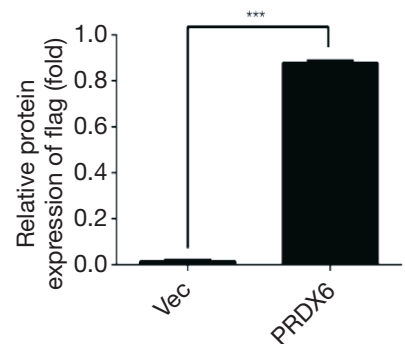

Toledo

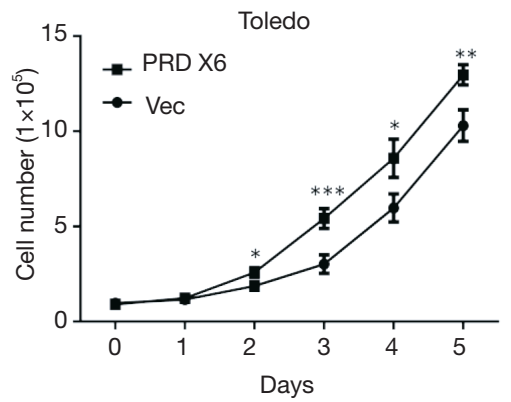

B

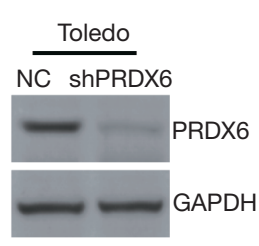

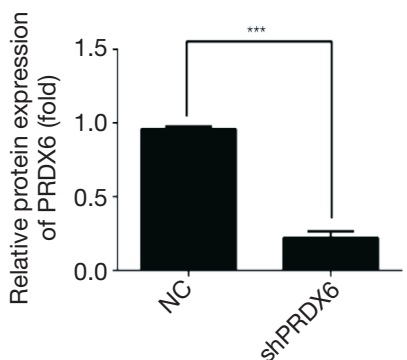

Toledo

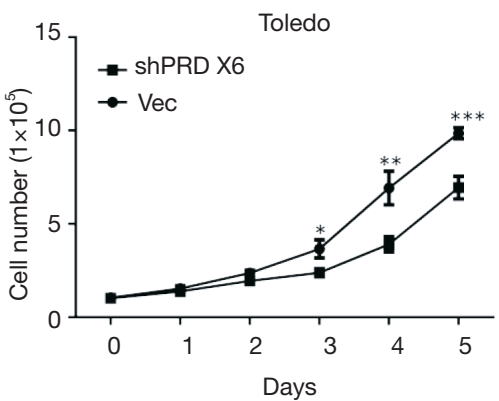

C
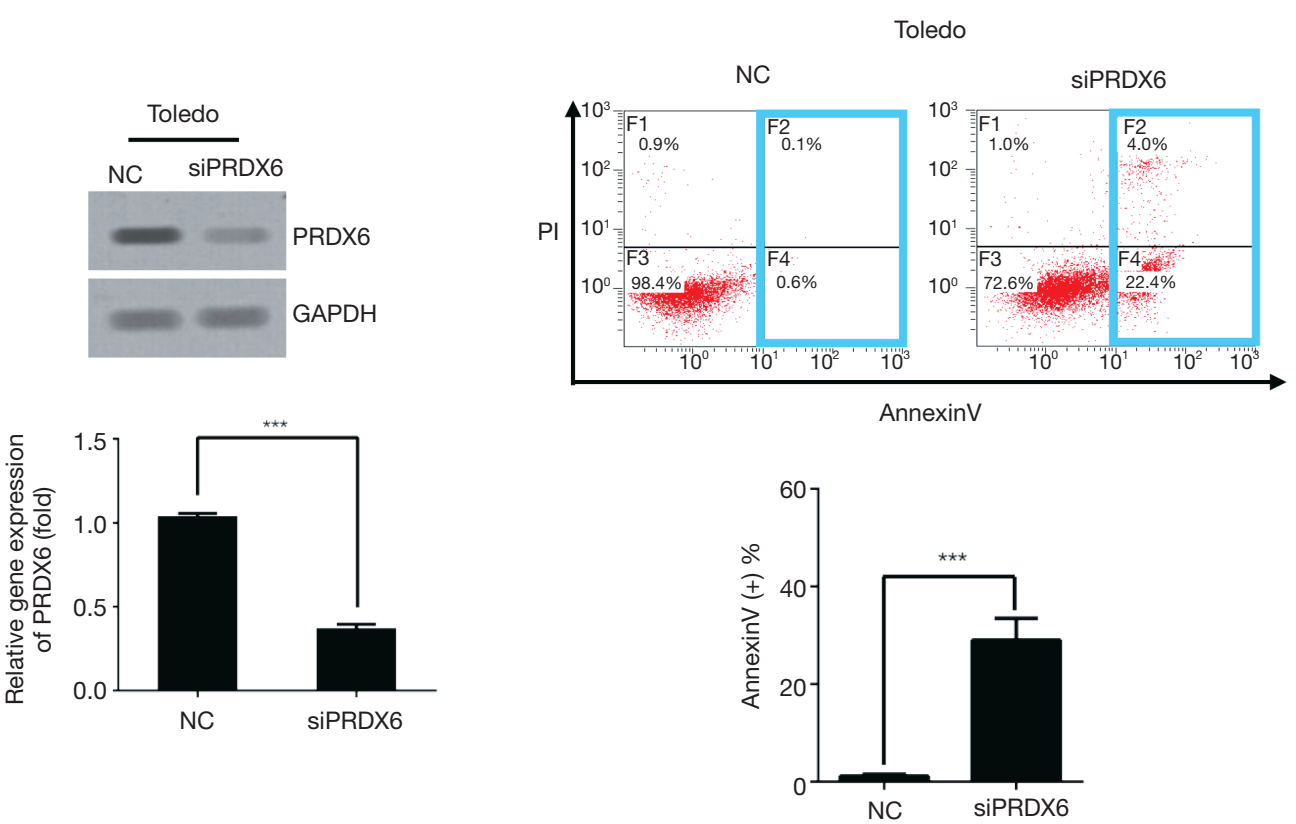

Figure 1 Effect of PRDX6 on Toledo DLBCL cells growth. (A) PRDX6-flag (PRDX6 cells) was stably expressed in Toledo cells by lentivirus mediated transfection. $3 \times 10^{6}$ of PRDX6 cells or control cells (Vec) were subjected for western analysis and relation protein quantification was showed. $1 \times 10^{5}$ of PRDX6 cells or control cells (Vec) were seeded in 6-well plate. For each day, three well of cell in each group were analyzed by cell counting assay. The data from three independent experiments were drawn a line graph. (B) PRDX6 shRNA (shPRDX6 cells) was stably expressed in Toledo cells by lentivirus mediated transfection. $3 \times 10^{6}$ of shPRDX6 cells or control cells (NC) were subjected for western analysis, and relation protein quantification was showed. $1 \times 10^{5}$ of shPRDX6 cells or control cells (NC) were seeded in 6-well plate. For each day, three well of cell in each group were analyzed by cell counting assay. The data from three independent experiments were drawn a line graph. (C) PRDX6 was knocked down by siRNA in Toledo cells $\left(3 \times 10^{6}\right)$. The Western blot was showed. (D) After 48h, cells knocked down by siRNA were collected for apoptosis analysis by testing with flow cytometry. The bar represents means \pm SD of three independent experiments $\left({ }^{*}, \mathrm{P}<0.05 ;{ }^{* *}, \mathrm{P}<0.01 ;{ }^{* * *} \mathrm{P},<0.001\right)$. DLBCL, diffuse large B cell lymphoma. 


\section{Downregulation of PRDX6 induces apoptosis in Toledo DLBCL cells}

We next evaluated the effect of PRDX6 on apoptosis. As shown in Figure S1, upregulation of PRDX6 did not induce apoptosis in Toledo DLBCL cells. However, downregulating the expression of PRDX6 by siRNA induced a substantial apoptosis in Toledo DLBCL cells (Figure 1C,D). The population of apoptotic cell were $28.97 \% \pm 3.70 \%(\mathrm{P}<0.001)$ in siPRDX6 cells and $1.13 \% \pm 0.33 \%$ in NC cells.

\section{PRDX6 induces chemo-resistance in Toledo DLBCL cells}

Chemo-resistance is the main obstacle faced in DLBCL treatment. We further detected whether PRDX6 might affect the sensitivity of DLBCL cells to doxorubicin, an important component in $\mathrm{R}-\mathrm{CHOP}$ regimen. As shown in Figure 2, $0.2 \mu \mathrm{M}$ doxorubicin treatment (Dox) significantly induced apoptosis in Toledo DLBCL cells (comparing the first and third panel of Figure $2 \mathrm{~A}$ up, comparing the first and third column of Figure $2 B$ left). The apoptosis populations in control group and Dox group were $2.07 \% \pm 0.41 \%$ and $49.90 \% \pm 2.74 \%(\mathrm{P}<0.001)$ respectively. However, overexpression of PRDX6 markedly reduced doxorubicin induced apoptosis (comparing the third and fourth panel of Figure $2 A$ up, comparing the third and fourth column of Figure $2 B$ left). The apoptosis populations in Dox group and Dox+ group were $49.90 \% \pm 2.74 \%$ and $16.07 \% \pm 1.83 \%$ $(\mathrm{P}<0.001)$ respectively.

\section{Downregulation of PRDX6 enhances Dox induced apoptosis in Toledo DLBCL cells}

We next examined the effect of PRDX6 downregulation on Dox induced apoptosis. As shown in Figure $2 A$ down and 2B right, we treated Toledo with $0.05 \mu \mathrm{M}$ doxorubicin to induce a moderate level of apoptosis. Low concentration of doxorubicin treatment induced $23.17 \% \pm 2.00 \%(\mathrm{P}<0.001)$ level of apoptosis as comparing with $2.36 \% \pm 0.63 \%$ in control group (comparing the first and third panel of Figure $2 A$ down, comparing the first and third column of Figure $2 B$ right). Downregulation of PRDX6 expression by siRNA also induced a moderate level of apoptosis $(23.17 \% \pm 2.00 \%)$. Interestingly, downregulation of PRDX6 expression substantially increased doxorubicin induced apoptosis (Figure $2 A$ down and $2 B$ right). The level of apoptosis in the combined group was $90.00 \% \pm 3.47 \%$ which was significantly higher than the apoptosis of Dox group plus siPRDX6 group (Figure $2 A$ down and Figure $2 B$ right). These data suggested that siPRDX6 might induce a synergetic apoptosis effect when combining with doxorubicin treatment.

\section{Peroxidase activity is responsible for PRDX6 induced cell growth and anti-apoptotic effects in Toledo DLBCL cells}

Since PRDX6 displays both glutathione peroxidase and phospholipase A2 (iPLA2) activities (4). We further examined which activity was responsible for PRDX6 induced proliferation and anti-apoptosis. We upregulated or downregulated PRDX6 expression in a dose-dependent manner by transient transfection. As shown in Figure $3 A, B$, the glutathione peroxidase and iPLA2 activities were gradually increased as the PRDX6 expression increasing. Conversely, the glutathione peroxidase and iPLA2 activities were gradually suppressed as the PRDX6 expression decreasing (Figure $3 C, D)$. We then inhibited the glutathione peroxidase or iPLA2 activities by M-succinate (mercaptosuccinate) or MJ33 respectively $(4,14)$. As shown in Figure 4A, M-succinate effectively reversed PRDX6 induced proliferation, but $\mathrm{MJ} 33$ did not. As shown in Figure 4B,C, PRDX6 alleviated Dox induced apoptosis. However, $M$-succinate, but not MJ33, overcame PRDX6 induced Dox resistance (Figure 4B,C). These data indicated that the glutathione peroxidase activity was critical for PRDX6 induced proliferation and Dox resistance.

\section{Discussion}

In the current study, we explored the function of PRDX6 in DLBCL cells. Our data showed PRDX6 is critical for the proliferation of Toledo DLBCL cells. Additionally, overexpression of PRDX6 conferred Dox-resistance to Toledo DLBCL cells. Moreover, combination of Dox treatment and siPRDX6 induced a synergetic effect on apoptosis. Mechanically, we showed that inhibition of glutathione peroxidase activity of PRDX6 by M-succinate, but not inhibition of phospholipase A2 activity of PRDX6 by MJ33, overcame PDRX6 induced proliferation and antiapoptosis effects.

DLBCL is a kind of aggressive lymphoma which composes of large, transformed B cells, and displays in diffuse growth pattern (15). The addition of rituximab to $\mathrm{CHOP}$ regimen generated a significant improvement in DLBCL treatment in two decades. Although the prognosis of DLBCL has been improved significantly, resistance 
A

Vec

PRDX6

Vec

PRDX6

Dox

Dox
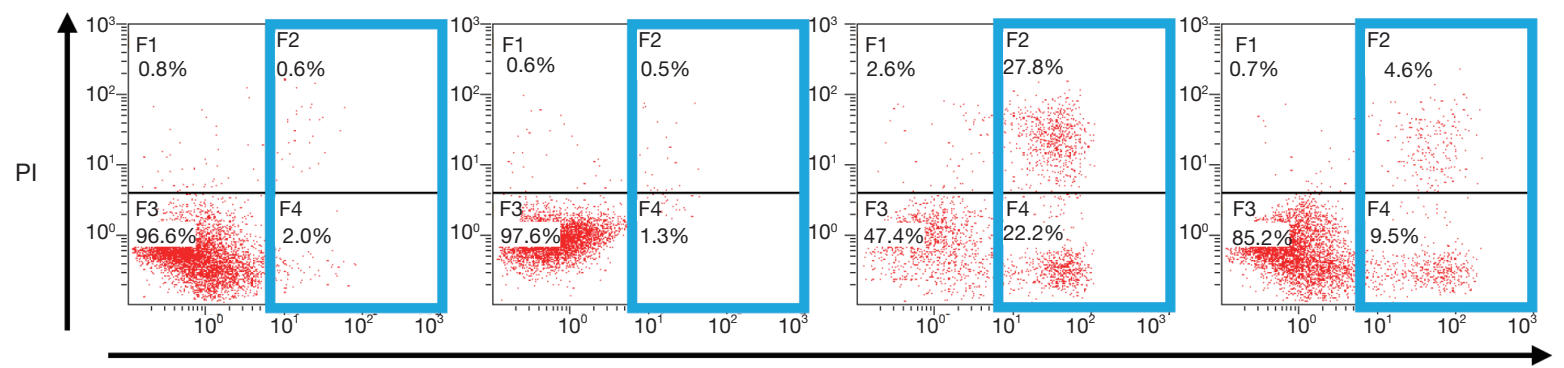

AnnexinV

NC

SiPRDX6

NC

SiPRDX6

Dox

Dox
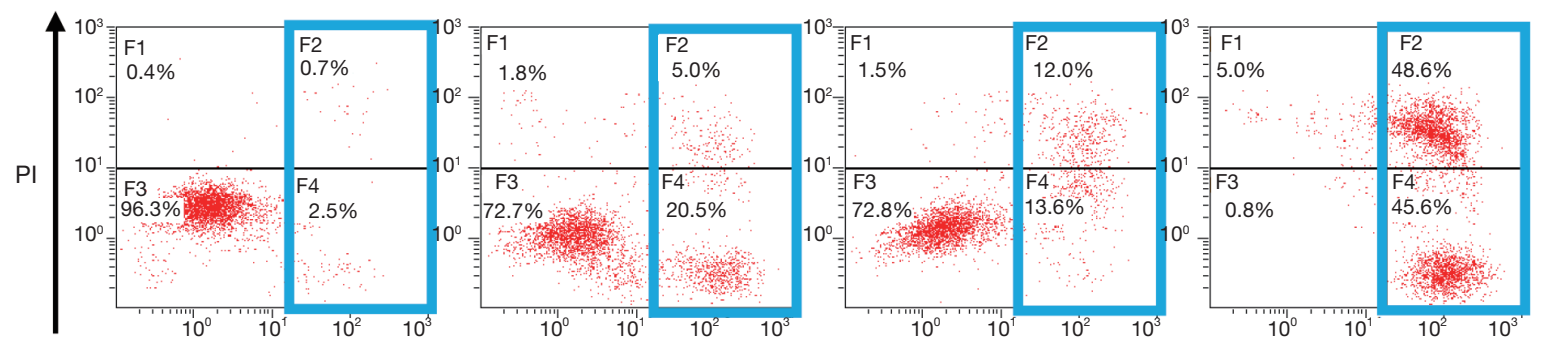

AnnexinV

B

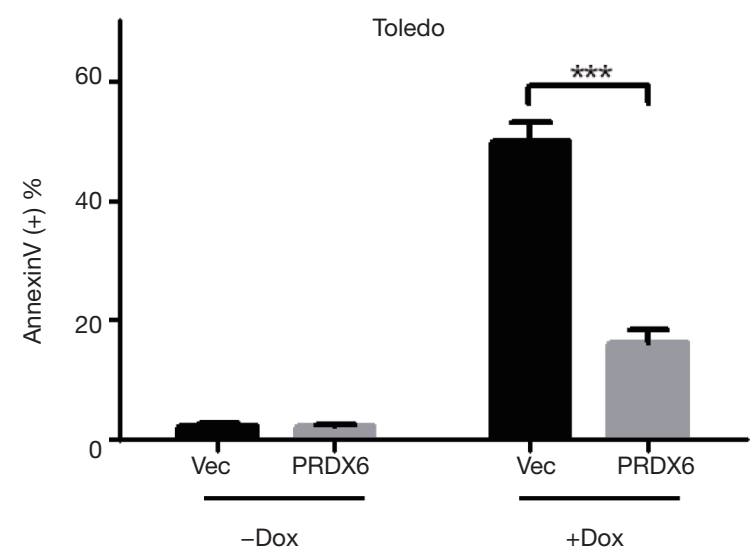

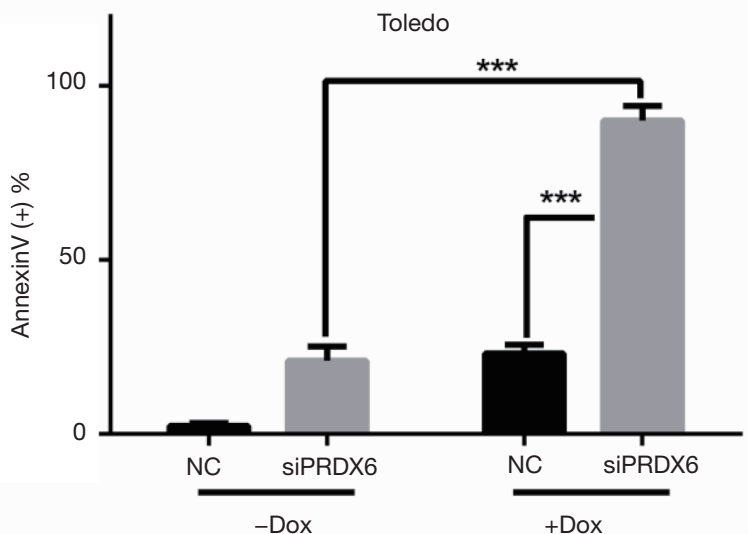

Figure 2 PRDX6 induces chemo-resistance and downregulation of PRDX6 enhances Dox induced apoptosis in Toledo DLBCL cells. (A) (up) Two sets of PRDX6 $\left(2 \times 10^{6}\right)$ cells and Vec cells $\left(2 \times 10^{6}\right)$ were seeded. One set of cells was treated with control reagent, the other set was treated with $0.2 \mu \mathrm{M}$ doxorubicin for $48 \mathrm{~h}$. Then, cells were collected for apoptosis analysis. (down) Four sets of Toledo $\left(2 \times 10^{6}\right)$ cells were seeded. These sets of cells were treated with control reagent plus non-specific siRNA, control reagent plus PRDX6 specific siRNA, $0.05 \mu M$ doxorubicin plus non-specific siRNA or $0.05 \mu \mathrm{M}$ doxorubicin plus PRDX6 specific siRNA for $48 \mathrm{~h}$. Then, cells were collected for apoptosis analysis. (B) The data from three independent experiments were drawn a histogram. The bar represents means \pm SD of three independent experiments $\left.{ }^{* * *}, \mathrm{P}<0.001\right)$. 
A

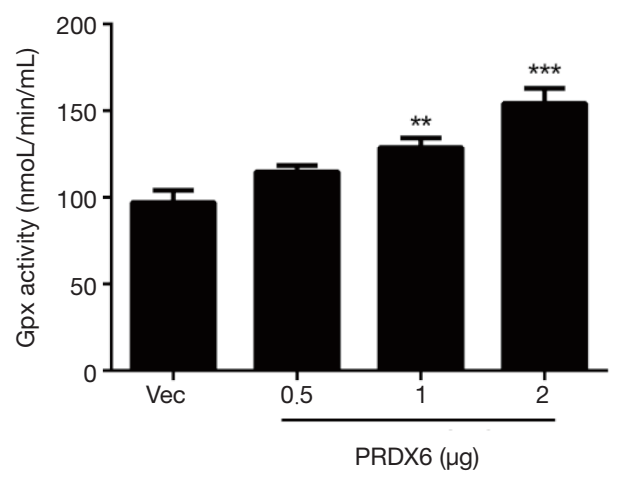

C

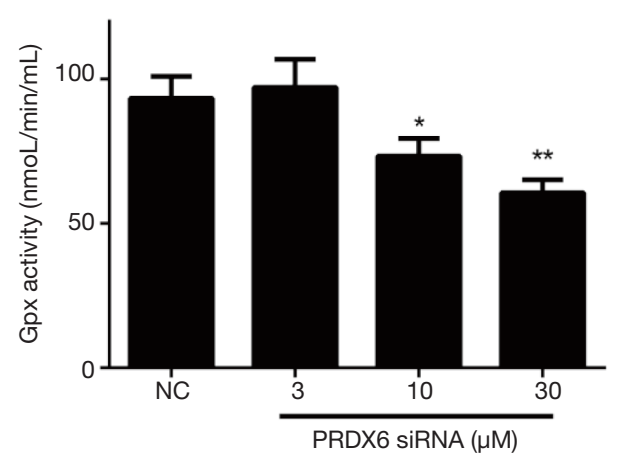

B

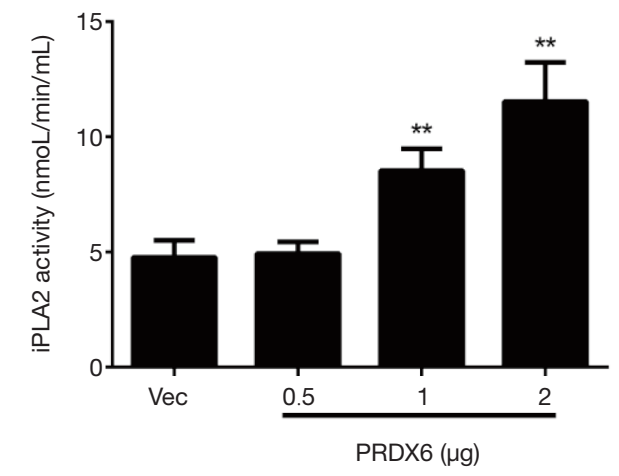

D

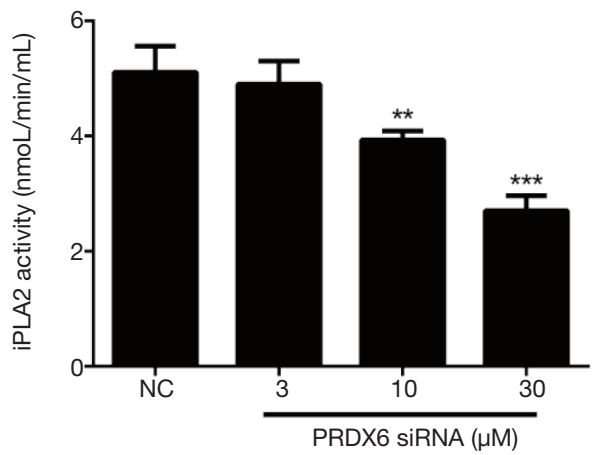

Figure 3 PRDX6 activates glutathione peroxidase and iPLA2 in Toledo DLBCL cells. (A and B). Toledo $\left(2 \times 10^{6}\right)$ cells were transiently transfected with indicated amount of plasmid expressed PRDX6-flag. After $48 \mathrm{~h}$, cells were collected for glutathione peroxidase (A) or iPLA2 activities analysis (B). (C and D) Toledo $\left(2 \times 10^{6}\right)$ cells were transiently transfected with indicated amount of non-specific siRNA or siRNA against PRDX6. After $48 \mathrm{~h}$, cells were collected for glutathione peroxidase (C) or iPLA2 activities analysis (D). DLBCL, diffuse large B cell lymphoma. ${ }^{*}, \mathrm{P}<0.05 ;{ }^{* *}, \mathrm{P}<0.01 ;{ }^{* * *}, \mathrm{P}<0.001$.

to the CHOP regimen continues to pose a problem in managing or curing DLBCL (16). Thus, elucidating the mechanism of chemo-resistance and identifying new therapeutic target are urgently needed to improve the quality of patient care and effectiveness of CHOP therapies. In this study, we focused on doxorubicin resistance in DLBCL, which is a main component of CHOP regimen. We showed that PRDX6 expression was critical for inducing doxorubicin resistance. Targeting PRDX6 not only overcame doxorubicin resistance, but also produced a synergetic effect on apoptosis in Toledo DLBCL cells.

PRDX6 is a member of the family of non-selenium thiol peroxidases. Recent studies have shown that PRDX6 are up-regulated in various cancers, including breast cancer (17), lung cancer (18) or tongue squamous cell carcinoma (19). However, there is no study about the function of PRDX6 in DLBCL. We studied the effects of PRDX6 on cell proliferation, apoptosis and drug resistance in DLBCL for the first time. Our data showed that the expression of PRDX6 was important for the cell proliferation and apoptosis in Toledo DLBCL cells. More importantly, PRDX6 was a crucial molecule mediated doxorubicin resistance. Targeting PRDX6 by siRNA produced synergetic effect on apoptosis when combined with doxorubicin treatment. These data suggested a promising therapeutic potential of PRDX6 in clinical treatment of DLBCL.

PRDX6 is a unique bifunctional enzyme in PRDX 


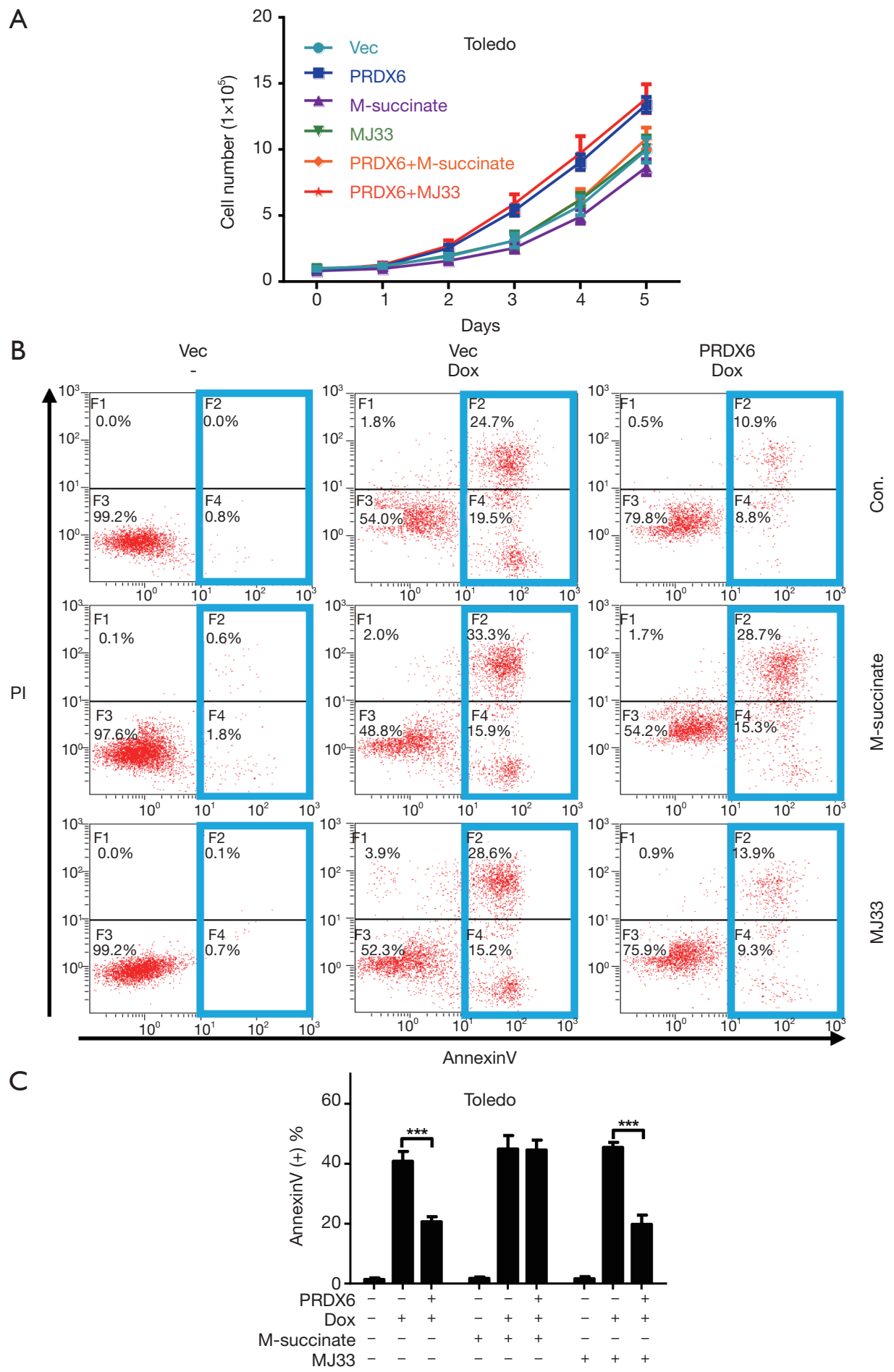

Figure 4 Peroxidase activity is responsible for PRDX6 induced cell growth and anti-apoptotic effects in Toledo DLBCL cells. (A) Three sets of PRDX6 $\left(2 \times 10^{6}\right)$ cells and Vec cells $\left(2 \times 10^{6}\right)$ were seeded. These sets of cells were treated with control reagent, $M$-succinate $(20 \mu M)$ or MJ33 $(10 \mu M)$ for indicated time. Cells were collected for cell number counting. The data from three independent experiments were drawn a line graph. (B) The experiment settings were similar with (A). After $48 \mathrm{~h}$, cells were collected for apoptosis analysis. The representative results were shown. (C) The data from three independent experiments of (B) were drawn a histogram. The bar represents means \pm SD of three independent experiments $\left.{ }^{* * *}, \mathrm{P}<0.001\right)$. 
family that contains, in addition to its peroxidase activity, iPLA2 activity (4). Previous studies indicated that the peroxidase activity and iPLA2 activity might have different function. For example, the peroxidase activity of PRDX6 was important for cell growth. This effect could be blocked by M-succinate, which was a peroxidase activity inhibitor, but not MJ33, which was a iPLA2 activity inhibitor (11). Conversely, the invasion ability was mainly supported by iPLA2 activity (11). Consistently, our study supported that PRDX6 promoted Toledo DLBCL cells proliferation via peroxidase activity. Importantly, we showed that the anti-apoptosis function of PRDX6 was also supported by peroxidase activity, but not iPLA2 activity. These data indicated that inhibition of PRDX6 peroxidase activity might also an effective strategy to overcome PRDX6 induced drug resistance in DLBCL.

Together, our study explored the tumor promoting function of PRDX6 in DLBCL. Our data implied that PRDX6 was a crucial factor to induce drug resistance. Targeting PRDX6 expression or peroxidase activity could be an effective strategy to overcome drug resistance in clinical DLBCL treatment.

\section{Acknowledgments}

Funding: This work was supported by the Natural Science Foundation of Guangdong Province (No. 2014A030310292 to L Liu).

\section{Footnote}

Conflicts of Interest: All authors have completed the ICMJE uniform disclosure form (available at http://dx.doi. org/10.21037/tcr.2019.08.36). The authors have no conflicts of interest to declare.

Ethical Statement: The authors are accountable for all aspects of the work in ensuring that questions related to the accuracy or integrity of any part of the work are appropriately investigated and resolved. This study was conducted in accordance with the Declaration of Helsinki (as revised in 2013). The institutional ethical approval and individual informed consent were waived.

Open Access Statement: This is an Open Access article distributed in accordance with the Creative Commons Attribution-NonCommercial-NoDerivs 4.0 International License (CC BY-NC-ND 4.0), which permits the non- commercial replication and distribution of the article with the strict proviso that no changes or edits are made and the original work is properly cited (including links to both the formal publication through the relevant DOI and the license). See: https://creativecommons.org/licenses/by-nc-nd/4.0/.

\section{References}

1. Siegel R, Ma J, Zou Z, et al. Cancer statistics, 2014. CA Cancer J Clin 2014;64:9-29.

2. Nakajima Y, Tomita N, Itabashi M, et al. Analysis of outcomes in patients with supra-diaphragmatic vs infradiaphragmatic diffuse large B cell lymphoma treated with R-CHOP therapy. Leuk Res 2015;39:198-203.

3. Ziepert M, Hasenclever D, Kuhnt E, et al. Standard International prognostic index remains a valid predictor of outcome for patients with aggressive CD20+ B-cell lymphoma in the rituximab era. J Clin Oncol 2010;28:2373-80.

4. Chen JW, Dodia C, Feinstein SI, et al. 1-Cys peroxiredoxin, a bifunctional enzyme with glutathione peroxidase and phospholipase A2 activities. J Biol Chem 2000;275:28421-7.

5. Manevich Y, Fisher AB. Peroxiredoxin 6, a 1-Cys peroxiredoxin, functions in antioxidant defense and lung phospholipid metabolism. Free Radic Biol Med 2005;38:1422-32.

6. Manevich Y, Sweitzer T, Pak JH, et al. 1-Cys peroxiredoxin overexpression protects cells against phospholipid peroxidation-mediated membrane damage. Proc Natl Acad Sci U S A 2002;99:11599-604.

7. Wang Y, Phelan SA, Manevich Y, et al. Transgenic mice overexpressing peroxiredoxin 6 show increased resistance to lung injury in hyperoxia. Am J Respir Cell Mol Biol 2006;34:481-6.

8. Hooks SB, Cummings BS. Role of $\mathrm{Ca} 2+-$ independent phospholipase A2 in cell growth and signaling. Biochem Pharmacol 2008;76:1059-67.

9. Walsh B, Pearl A, Suchy S, et al. Overexpression of Prdx6 and resistance to peroxide-induced death in Hepa1-6 cells: Prdx suppression increases apoptosis. Redox Rep 2009; 14:275-84.

10. Schmitt A, Schmitz W, Hufnagel A, et al. Peroxiredoxin 6 triggers melanoma cell growth by increasing arachidonic acid-dependent lipid signalling. Biochem J 2015;471:267-79.

11. Ho JN, Lee SB, Lee SS, et al. Phospholipase A2 activity of peroxiredoxin 6 promotes invasion and metastasis of lung cancer cells. Mol Cancer Ther. 2010;9:825-32. 
12. Machado-Neto JA, Coelho-Silva JL, Santos FPS, et al. Autophagy inhibition potentiates ruxolitinib-induced apoptosis in JAK2V617F cells. Invest New Drugs 2019. [Epub ahead of print].

13. Yun HM, Park KR, Lee HP, et al. PRDX6 promotes lung tumor progression via its GPx and iPLA2 activities. Free Radic Biol Med 2014;69:367-76.

14. Kim TS, Sundaresh CS, Feinstein SI, et al. Identification of a human cDNA clone for lysosomal type $\mathrm{Ca} 2+-$ independent phospholipase $\mathrm{A} 2$ and properties of the expressed protein. J Biol Chem 1997;272:2542-50.

15. Chapuy B, Stewart C, Dunford AJ, et al. Molecular subtypes of diffuse large B cell lymphoma are associated with distinct pathogenic mechanisms and outcomes. Nat Med 2018;24:679-90.

Cite this article as: Fang Z, Liu T, Liu X, Lu Y, Sun Y, Xiao R, Fan R, Liu L. PRDX6 promotes proliferation and induces chemo-resistance via peroxidase activity in Toledo diffuse large B-cell lymphoma cells. Transl Cancer Res 2019;8(5):1772-1781. doi: $10.21037 /$ tcr.2019.08.36
16. Lazzarino M, Orlandi E, Paulli M, et al. Treatment outcome and prognostic factors for primary mediastinal (thymic) B-cell lymphoma: a multicenter study of 106 patients. J Clin Oncol 1997;15:1646-53.

17. Liu FJ, Wang XB, Cao AG. Screening and functional analysis of a differential protein profile of human breast cancer. Oncol Lett 2014;7:1851-6.

18. Rostila A, Puustinen A, Toljamo T, et al. Peroxiredoxins and tropomyosins as plasma biomarkers for lung cancer and asbestos exposure. Lung Cancer 2012;77:450-9.

19. Yanagawa T, Omura K, Harada H, et al. Peroxiredoxin I expression in tongue squamous cell carcinomas as involved in tumor recurrence. Int J Oral Maxillofac Surg 2005;34:915-20. 


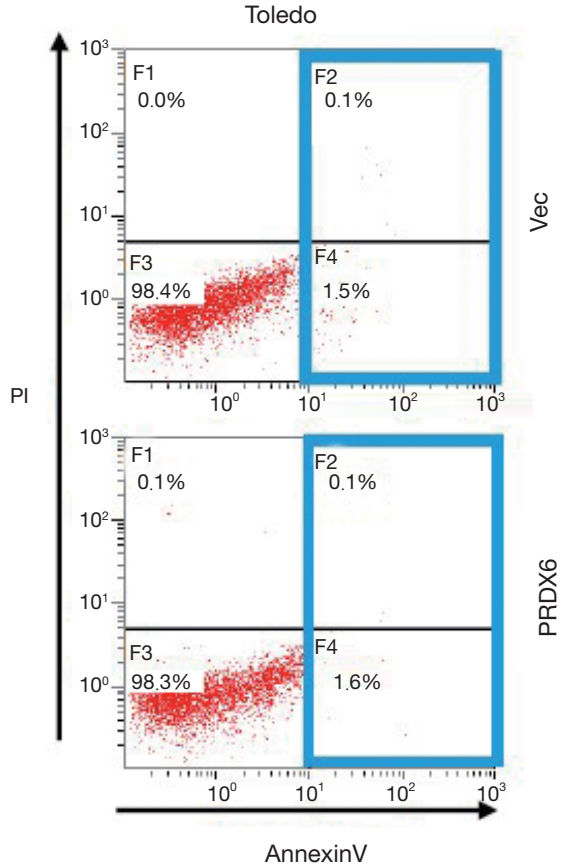

Figure S1 Upregulation of PRDX6 does not induce apoptosis in Toledo DLBCL cells. DLBCL, diffuse large B cell lymphoma. 